Pacific

Journal of

Mathematics

\title{
QUASICONFORMAL HARMONIC MAPPINGS BETWEEN DINI-SMOOTH JORDAN DOMAINS
}

DAVID KALAJ 


\title{
QUASICONFORMAL HARMONIC MAPPINGS BETWEEN DINI-SMOOTH JORDAN DOMAINS
}

\author{
DAVID KALAJ
}

\begin{abstract}
Let $D$ and $\Omega$ be Jordan domains with Dini-smooth boundaries. We prove that if $f: D \rightarrow \Omega$ is a harmonic homeomorphism and $f$ is quasiconformal, then $f$ is Lipschitz. This extends some recent results, where stronger assumptions on the boundary are imposed. Our result is optimal in that it coincides with the best condition for Lipschitz behavior of conformal mappings in the plane and conformal parametrizations of minimal surfaces.
\end{abstract}

\section{Introduction and statement of the main result}

Quasiconformal mappings. By definition, $K$-quasiconformal mappings (or qc mappings for short) are orientation-preserving homeomorphisms $f: D \rightarrow \Omega$ between domains $D, \Omega \subset \mathbb{C}$ that are contained in the Sobolev class $W_{\text {loc }}^{1,2}(D)$ and for which the differential matrix and its determinant are coupled in the distortion inequality

$$
|D f(z)|^{2} \leq K \operatorname{det} D f(z), \quad \text { where }|D f(z)|=\max _{|\xi|=1}|D f(z) \xi|,
$$

for some $K \geq 1$. Here det $D f(z)$ is the determinant of the formal derivative $D f(z)$, which will be denoted in the sequel by $J_{f}(z)$. Note that condition (1-1) can be written in complex notation as

$$
\left(\left|f_{z}\right|+\left|f_{\bar{z}}\right|\right)^{2} \leq K\left(\left|f_{z}\right|^{2}-\left|f_{\bar{z}}^{2}\right|\right) \quad \text { a.e. on } D,
$$

or, what is the same,

$$
\left|f_{\bar{z}}\right| \leq k\left|f_{z}\right| \quad \text { a.e. on } D, \quad \text { where } k=\frac{K-1}{K+1} \text {, i.e., } K=\frac{1+k}{1-k} .
$$

Harmonic mappings and the Hilbert transform. A mapping $f$ is called harmonic in a region $D$ if it has the form $f=u+i v$, where $u$ and $v$ are real-valued harmonic functions in $D$. If $D$ is simply connected, then there are two analytic functions $h$ and $g$ defined on $D$ such that $f$ has the representation

$$
f=h+\bar{g} .
$$

MSC2010: primary 30C55; secondary 31C05.

Keywords: planar harmonic mappings, quasiconformal, Dini-smooth. 
If $f$ is a harmonic univalent function, then by Lewy's theorem [1936], $f$ has a nonvanishing Jacobian and therefore is a diffeomorphism by the inverse mapping theorem.

Let

$$
P(r, x-\varphi)=\frac{1-r^{2}}{2 \pi\left(1-2 r \cos (x-\varphi)+r^{2}\right)}
$$

denote the Poisson kernel. If $F \in L^{1}(\mathbb{T})$, where $\mathbb{T}$ is the unit circle, we define the Poisson integral $\mathcal{P}[F]$ of $F$ by

$$
\mathcal{P}[F](z)=\int_{0}^{2 \pi} P(r, x-\varphi) F\left(e^{i x}\right) d x, \quad|z|<1, z=r e^{i \varphi} .
$$

The function $f(z)=\mathcal{P}[F](z)$ is a harmonic mapping in the unit disk $\mathbb{U}=\{z:|z|<1\}$, which belongs to the Hardy space $h^{1}(\mathbb{U})$. The mapping $f$ is bounded in $\mathbb{U}$ if and only if $F \in L^{\infty}(\mathbb{T})$. Standard properties of the Poisson integral show that $\mathcal{P}[F]$ extends by continuity to $F$ on $\overline{\mathbb{U}}$, provided that $F$ is continuous. For these facts and standard properties of harmonic Hardy spaces, we refer to [Axler et al. 1992, Chapter 6; Duren 1970]. With the additional assumption that $F$ is an orientationpreserving homeomorphism of this circle onto a convex Jordan curve $\gamma, \mathcal{P}[F]$ is an orientation-preserving diffeomorphism of the open unit disk onto the region bounded by $\gamma$. This is indeed the celebrated theorem of Choquet-Radó-Kneser [Choquet 1945; Duren 2004]. This theorem is not true for nonconvex domains, but does hold under some additional assumptions. It has been extended in various directions (see for example [Jost 1981; Kalaj 2011b; Duren and Hengartner 1997]).

If $f=u+i v$ is a harmonic function defined in a Dini-smooth Jordan domain $D$ then a harmonic function $\tilde{f}=\tilde{u}+i \tilde{v}$ is called the harmonic conjugate of $f$ if $u+i \tilde{u}$ and $v+i \tilde{v}$ are analytic functions. Notice that $\tilde{f}$ is uniquely determined up to an additive constant. Let $\Phi: D \rightarrow \mathbb{U}$ be a conformal mapping, and let $G \in L^{1}(\partial D)$. Then the Poisson integral of $G$ with respect to the domain $D$ is defined by

$$
\mathcal{P}_{D}[G](z)=\frac{1}{2 \pi} \int_{\partial D} \frac{1-|\Phi(z)|^{2}}{|\Phi(z)-\Phi(\zeta)|^{2}} G(\zeta)\left|\Phi^{\prime}(\zeta)\right| d \zeta .
$$

Let $\chi$ be the boundary value of $f$ and assume that $\tilde{\chi}$ is the boundary value of $\tilde{f}$. Then $\tilde{\chi}$ is called the Hilbert transform of $\chi$ and we also write it as $H(\chi)$. Assume that $\tilde{\chi} \in L^{1}(\partial D)$. In particular, the Hilbert transform of a function $\chi \in L^{1}(\mathbb{T})$ is defined by the formula

$$
\tilde{\chi}(\tau)=H(\chi)(\tau)=-\frac{1}{\pi} \int_{0^{+}}^{\pi} \frac{\chi(\tau+t)-\chi(\tau-t)}{2 \tan (t / 2)} d t .
$$

Here $\int_{0^{+}}^{\pi} \Phi(t) d t:=\lim _{\epsilon \rightarrow 0^{+}} \int_{\epsilon}^{\pi} \Phi(t) d t$. This integral is improper and converges for a.e. $\tau \in[0,2 \pi]$. This and other facts concerning the operator $H$ used in this 
paper can be found in [Zygmund 1959, Chapter VII]. Assume that $\chi, \tilde{\chi}$ are in $L^{1}(\mathbb{T})$. Then

$$
\mathcal{P}[\tilde{\chi}]=(\mathcal{P}[\chi])^{\sim},
$$

where $(k)^{\sim}$ is the harmonic conjugate of $k$ (see for instance [Pavlović 2004, Theorem 6.1.3]).

If $f=h+\bar{g}: \mathbb{U} \rightarrow \Omega$ is a harmonic mapping then the radial and tangential derivatives at $z=r e^{i t}$ are defined by

$$
\partial_{r} f(z)=\frac{1}{r}\left(h^{\prime}+\bar{g}^{\prime}\right) \quad \text { and } \quad \partial_{t} f(z)=i\left(h^{\prime}-\bar{g}^{\prime}\right) .
$$

So $r \partial_{r} f$ is the harmonic conjugate of $\partial_{t} f$. We generalize this definition for a mapping $f=h+\bar{g}$ defined in a Jordan domain $D$. In order to do so, let $\Phi=R e^{i \Theta}$ be a conformal mapping of the domain $D$ onto the unit disk. Then the radial derivative and tangent derivative of $f$ in a point $w \in D$ are defined by

$$
\partial_{R} f(w)=\frac{1}{|\Phi(w)|} D f(w)\left(\frac{\Phi(w)}{\Phi^{\prime}(w)}\right) \quad \text { and } \quad \partial_{\Theta} f(w)=D f(w)\left(i \frac{\Phi(w)}{\Phi^{\prime}(w)}\right) .
$$

Here $\Phi(w) / \Phi^{\prime}(w)$ and $i\left(\Phi(w) / \Phi^{\prime}(w)\right)$ are treated as two vectors from $\mathbb{R}^{2} \cong \mathbb{C}$. Then it is easy to show that

$$
R \partial_{R} f(w)=\frac{h^{\prime}(w)}{\Phi^{\prime}(w)}+\frac{\overline{g^{\prime}(w)}}{\overline{\Phi^{\prime}(w)}} \quad \text { and } \quad \partial_{\Theta} f(w)=i\left(\frac{h^{\prime}(w)}{\Phi^{\prime}(w)}-\frac{\overline{g^{\prime}(w)}}{\overline{\Phi^{\prime}(w)}}\right) .
$$

This implies that $R \partial_{R} f(w)$ and $\partial_{\Theta} f(w)$ are harmonic functions in $D$ and $R \partial_{R} f(w)$ is the harmonic conjugate of $\partial_{\Theta} f(w)$. Notice also that these derivatives are uniquely determined up to a conformal mapping $\Phi$. Assume further that $D$ and $\Omega$ have Dini-smooth boundaries. If $F: \partial D \rightarrow \partial \Omega$ is the boundary function of $f$, and if $\partial_{\Theta} f(w)$ is a bounded harmonic function, then

$$
\lim _{w \rightarrow w_{0}} \partial_{\Theta} f(w)=F^{\prime}\left(w_{0}\right),
$$

where the limit is nontangential. Here

$$
F^{\prime}\left(w_{0}\right):=\frac{\partial\left(F \circ \Phi^{-1}\right)\left(e^{i t}\right)}{\partial t},
$$

where $\Phi\left(w_{0}\right)=e^{i t}$. If $F^{\prime} \in L^{1}(\partial D)$, then the harmonic function $R \partial_{R} f(w)$ has nontangential limits in almost every point of $\partial D$ and its boundary value is the Hilbert transform of $F^{\prime}$, namely

$$
H\left(F^{\prime}\right)\left(w_{0}\right)=\lim _{w \rightarrow w_{0}} R \partial_{R} f(w) .
$$


From now on the boundary value of $f$ will be denoted by $F$. We will focus on orientation-preserving harmonic quasiconformal mappings between smooth domains and investigate their Lipschitz character up to the boundary. For future reference, we will say that a qc mapping $f: \mathbb{U} \rightarrow \Omega$ of the unit disk onto the Jordan domain $\Omega$ with rectifiable boundary is normalized if $f(1)=w_{0}, f\left(e^{2 \pi i / 3}\right)=w_{1}$ and $f\left(e^{4 \pi i / 3}\right)=w_{2}$, where $w_{0} w_{1}, w_{1} w_{2}$ and $w_{2} w_{0}$ are arcs of $\gamma=\partial \Omega$ having the same length $|\gamma| / 3$.

Background. Let $\Omega$ be a Jordan domain with rectifiable boundary, and let $\gamma$ be an arc-length parametrization of $\partial \Omega$. We say that $\partial \Omega$ is $C^{1}$ if $\gamma \in C^{1}$. Then $\arg \gamma^{\prime}$ is continuous and we let $\omega$ be its modulus of continuity. If $\omega$ satisfies

$$
\int_{0}^{\delta} \frac{\omega(t)}{t} d t<\infty, \quad \delta>0
$$

we say that $\partial \Omega$ is Dini-smooth. Denote by $C^{1, \varpi}$ the class of all Dini-smooth Jordan curves. The derivative of a conformal mapping $f$ of the unit disk onto $\Omega$ is continuous and nonvanishing in $\bar{D}$ [Pommerenke 1975, Theorem 10.2] (see also [Warschawski 1961]). This implies that $f$ is bi-Lipschitz continuous. For later reference we refer to this result as Kellogg's theorem, see [Kellogg 1912; Goluzin 1969, p. 374]. Kellogg was the first to consider this type of result for $C^{1, \alpha}$ domains, where $0<\alpha<1$. Warschawski [1970] proved the same result for a conformal parametrization of a minimal surface.

If $f$ is merely quasiconformal and maps the unit disk onto itself, then Mori's theorem implies that $|f(z)-f(w)| \leq M_{1}(K)|z-w|^{1 / K}$. The constant $1 / K$ is the best possible. If $f$ is a conformal mapping of the unit disk onto a Jordan domain with a $C^{1}$ boundary, then the function $f$ is not necessarily Lipschitz (see for example [Lesley and Warschawski 1978, p. 277]). This is why we need to add some assumption, other than quasiconformality, as well as some smoothness of the image curve that is better than $C^{1}$ in order to obtain that the resulting mapping is Lipschitz or bi-Lipschitz.

Since every conformal mapping in the plane is harmonic and quasiconformal, it is an interesting question to ask to what extent the smoothness of the boundary of a Jordan domain $\Omega$ implies that a quasiconformal harmonic mapping of the unit disk onto $\Omega$ is Lipschitz. The first study of harmonic quasiconformal mappings of the unit disk onto itself was done by O. Martio [1968]. This paper has been generalized in [Kalaj 2004] for qc mappings from the unit disk onto a convex Jordan domain. Pavlović [2002] proved in a very interesting way that every qc harmonic mapping of the unit disk onto itself is Lipschitz. Kalaj [2008] proved that every qc harmonic mapping between two Jordan domains with $C^{1, \alpha}$ boundary is Lipschitz. This result has its counterpart for non-Euclidean metrics [Kalaj and Mateljević 2006]. For a 
generalization of the last result to the several-dimensional case we refer to [Kalaj 2013]. The problem of bi-Lipschitz continuity of a quasiconformal mapping of the unit disk onto a Jordan domain with $C^{2}$ boundary has been solved in [Kalaj 2011a]. The object of this paper is to extend some of these results.

New results. The following theorem is such an extension in which the Hölder continuity is replaced by the more general Dini condition.

Theorem 1.1. Let $f=\mathcal{P}[F](z)$ be a harmonic normalized $K$-quasiconformal mapping between the unit disk and the Jordan domain $\Omega$ with $\gamma=\partial \Omega \in C^{1, \varpi}$. Then there exists a constant $C^{\prime}=C^{\prime}(\gamma, K)$ such that

$$
\left|\frac{\partial F\left(e^{i \varphi}\right)}{\partial \varphi}\right| \leq C^{\prime} \quad \text { for almost every } \varphi \in[0,2 \pi],
$$

and

$$
\left|f\left(z_{1}\right)-f\left(z_{2}\right)\right| \leq K C^{\prime}\left|z_{1}-z_{2}\right| \quad \text { for } z_{1}, z_{2} \in \mathbb{U} .
$$

By using Theorem 1.1, we obtain the following improvement of [Kalaj 2008, Theorem 3.1].

Theorem 1.2. Let $D$ and $\Omega$ be Jordan domains such that $\partial D$ and $\partial \Omega$ are contained in $C^{1, \varpi}$ and let $f: D \mapsto \Omega$ be a harmonic homeomorphism. The following statements hold true.

(a) If $f$ is qc, then $f$ is Lipschitz.

(b) If $\Omega$ is convex and $f$ is $q c$, then $f$ is bi-Lipschitz.

(c) If $\Omega$ is convex, then $f$ is $q c$ if and only $\log \left|F^{\prime}\right|$ and $H\left(F^{\prime}\right)$ are in $L^{\infty}(\partial D)$.

Proof of Theorem 1.2. (a) Choose a conformal mapping $\Phi: \mathbb{U} \rightarrow D$ so that the qc mapping $f_{1}=f \circ \Phi$ is normalized. Then $f_{1}$ is a qc harmonic mapping of the unit disk onto $\Omega$ that satisfies the conditions of Theorem 1.1. This implies in particular that $f_{1}$ is Lipschitz. In view of Kellogg's theorem, the mapping $\Phi$ is bi-Lipschitz. Thus $f=f_{1} \circ \Phi^{-1}$ is Lipschitz.

(b) If $\Omega$ is a convex domain, and if $D=\mathbb{U}$, then by [Kalaj 2003], we have that

$$
|D f(z)| \geq \frac{1}{4} \operatorname{dist}(f(0), \partial \Omega)
$$

for $z \in \mathbb{U}$. If $D$ is not the unit disk, then we make use of the conformal mapping $\Phi: \mathbb{U} \rightarrow D$ as in the proof of (a). Then we obtain

$$
|D f(z)|=\left|D f_{1}(z)\right| /\left|\Phi^{\prime}(z)\right| \geq c .
$$

Now by using the quasiconformality of $f$, we have that

$$
|D f(z)|^{2} \leq K J_{f}(z) .
$$


Therefore

$$
J_{f^{-1}(f(z))}=\frac{1}{J_{f}(z)} \leq \frac{K}{c^{2}} .
$$

Since $f^{-1}$ is $K$-quasiconformal, we have further that

$$
\left|D f^{-1}(w)\right|^{2} \leq K J_{f^{-1}}(w) \leq \frac{K^{2}}{c^{2}} .
$$

This implies that $f^{-1}$ is Lipschitz. This finishes the proof of (b).

(c) If $f$ is harmonic and quasiconformal, then by (b) it is bi-Lipschitz, and so its boundary function $F$ is bi-Lipschitz. Furthermore, $R \partial_{R} f$ is a bounded harmonic function and this is equivalent with the fact that $\log \left|F^{\prime}\right| \in L^{\infty}(\partial D)$. Since $H\left(F^{\prime}\right)$ is its boundary function, it is bounded, i.e., it belongs to $L^{\infty}(\partial D)$.

We now prove the opposite implication. Since

$$
\partial_{\Theta} f=\mathcal{P}_{D}\left[F^{\prime}\right] \quad \text { and } \quad R \partial_{R} f=\mathcal{P}_{D}\left[H\left(F^{\prime}\right)\right],
$$

it follows that $\partial_{\Theta} f$ and $R \partial_{R} f$ are bounded harmonic functions. This means that $|D f|$ is bounded by a constant $M$. In order to show that $f$ is quasiconformal, it is enough to show that the Jacobian of $f$ is bigger than a positive constant in $D$. Let $f_{1}=f \circ \Phi^{-1}$, and let $\delta=\operatorname{dist}\left(f_{1}(0), \partial \Omega\right)$ and $\kappa=\min \left|\partial_{t} f_{1}\left(e^{i t}\right)\right|$. Then by [Kalaj 2004, Corollary 2.9], we have

$$
J_{f}(\Phi(w))\left|\Phi^{\prime}(w)\right|^{2}=J_{f_{1}}(w) \geq \frac{\kappa \delta}{2} .
$$

So

$$
J_{f}(z) \geq c>0, \quad z \in D .
$$

We conclude that

$$
\frac{|D f(z)|^{2}}{J_{f}(z)} \leq \frac{M^{2}}{c}
$$

\section{Preliminary results}

Definition 2.1. Let $\xi:[a, b] \rightarrow \mathbb{C}$ be a continuous function. The modulus of continuity of $\xi$ is

$$
\omega(t)=\omega_{\xi}(t)=\sup _{|x-y| \leq t}|\xi(x)-\xi(y)| .
$$

The function $\xi$ is called Dini-continuous if

$$
\int_{0}^{b-a} \frac{\omega_{\xi}(t)}{t} d t<\infty
$$

Let $\gamma$ be a $C^{1}$ Jordan curve $\gamma$ with the length $l=|\gamma|$ and assume that $g:[0, l] \rightarrow \gamma$ is its arc-length parametrization. We say that $\gamma$ is Dini-smooth if $g^{\prime}$ is Dini-continuous 
on $[0, l]$. If $\omega(t)$ is the modulus of continuity of $g^{\prime}$ for $0 \leq t \leq l$, then we extend $\omega$ by $\omega(t)=\omega(l)$ for $t \geq l$.

A function $F: \mathbb{T} \rightarrow \gamma$ is called Dini-smooth if the function $\Phi(t)=F\left(e^{i t}\right)$ is Dini-smooth, i.e.,

$$
\left|\Phi^{\prime}(t)-\Phi^{\prime}(s)\right| \leq \omega(|t-s|),
$$

where $\omega$ is Dini-continuous. Observe that every smooth $C^{1, \alpha}$ Jordan curve is Dini-smooth.

Let

$$
\mathcal{K}(s, t)=\operatorname{Re}\left[\overline{(g(t)-g(s))} \cdot i g^{\prime}(s)\right]
$$

be a function defined on $[0, l] \times[0, l]$. By $\mathcal{K}(s \pm l, t \pm l)=\mathcal{K}(s, t)$ we extend it to $\mathbb{R} \times \mathbb{R}$. Suppose now that $\Psi: \mathbb{R} \mapsto \gamma$ is an arbitrary $2 \pi$-periodic Lipschitz function such that $\left.\Psi\right|_{[0,2 \pi)}:[0,2 \pi) \mapsto \gamma$ is an orientation-preserving bijective function. Then there exists an increasing continuous function $\psi:[0,2 \pi] \mapsto[0, l]$ such that

$$
\Psi(\tau)=g(\psi(\tau)) .
$$

We have for a.e. $e^{i \tau} \in \mathbb{T}$ that

$$
\Psi^{\prime}(\tau)=g^{\prime}(\psi(\tau)) \cdot \psi^{\prime}(\tau),
$$

and therefore

$$
\left|\Psi^{\prime}(\tau)\right|=\left|g^{\prime}(\psi(\tau))\right| \cdot\left|\psi^{\prime}(\tau)\right|=\psi^{\prime}(\tau) .
$$

Along with the function $\mathcal{K}$ we will also consider the function $\mathcal{K}_{F}$ defined by

$$
\mathcal{K}_{F}(t, \tau)=\operatorname{Re}\left[\overline{(\Psi(t)-\Psi(\tau))} \cdot i \Psi^{\prime}(\tau)\right] .
$$

Here $F\left(e^{i t}\right)=\Psi(t)$. It is easy to see that

$$
\mathcal{K}_{F}(t, \tau)=\psi^{\prime}(\tau) \mathcal{K}(\psi(t), \psi(\tau)) .
$$

Lemma 2.2. Let $\gamma$ be a Dini-smooth Jordan curve and let $g:[0, l] \mapsto \gamma$ be a natural parametrization of a Jordan curve with $g^{\prime}$ having modulus of continuity $\omega$. Assume further that $\Psi:[0,2 \pi] \mapsto \gamma$ is an arbitrary parametrization of $\gamma$ and let $F\left(e^{i t}\right)=\Psi(t)$. Then

$$
|\mathcal{K}(s, t)| \leq \int_{0}^{\min \{|s-t|, l-|s-t|\}} \omega(\tau) d \tau
$$

and

$$
\left|\mathcal{K}_{F}(\varphi, x)\right| \leq\left|\psi^{\prime}(\varphi)\right| \int_{0}^{d_{\gamma}(\Psi(\varphi), \Psi(x))} \omega(\tau) d \tau .
$$

Here $d_{\gamma}(\Psi(\varphi), \Psi(x)):=\min \{|s(\varphi)-s(x)|,(l-|s(\varphi)-s(x)|)\}$ is the (shortest) 
distance between $\Psi(\varphi)$ and $\Psi(x)$ along $\gamma$, and it satisfies

$$
|\Psi(\varphi)-\Psi(x)| \leq d_{\gamma}(\Psi(\varphi), \Psi(x)) \leq B_{\gamma}|\Psi(\varphi)-\Psi(x)| .
$$

Proof. Note that the estimate (2-5) has been proved in [Kalaj 2011b, Lemma 2.3]. Now (2-6) follows from (2-5) and (2-4).

A closed rectifiable Jordan curve $\gamma$ satisfies a $B$-chord-arc condition for some constant $B>1$ if for all $z_{1}, z_{2} \in \gamma$ we have

$$
d_{\gamma}\left(z_{1}, z_{2}\right) \leq B\left|z_{1}-z_{2}\right| .
$$

Here $d_{\gamma}\left(z_{1}, z_{2}\right)$ is the length of the shorter arc of $\gamma$ with endpoints $z_{1}$ and $z_{2}$. It is clear that if $\gamma \in C^{1}$, then $\gamma$ satisfies a chord-arc condition for some $B_{\gamma}>1$. The following lemma is proved in [Kalaj 2012].

Lemma 2.3. Assume that $\gamma$ satisfies a chord-arc condition for some $B>1$. Then for every normalized $K$-qc mapping $f$ between the unit disk $\mathbb{U}$ and the Jordan domain $\Omega=$ int $\gamma$ we have

$$
\left|f\left(z_{1}\right)-f\left(z_{2}\right)\right| \leq \Lambda_{\gamma}(K)\left|z_{1}-z_{2}\right|^{\alpha}, \quad z_{1}, z_{2} \in \mathbb{T},
$$

where

$$
\alpha=\frac{2}{K(1+2 B)^{2}}, \quad \Lambda_{\gamma}(K)=4 \cdot 2^{\alpha}(1+2 B) \sqrt{\frac{2 \pi K|\Omega|}{\log 2}} .
$$

Next we recall some estimates for the Jacobian of a harmonic univalent function.

Lemma 2.4 [Kalaj 2011b, Lemma 3.1]. Suppose $f=\mathcal{P}[F]$ is a harmonic mapping such that $F$ is a Lipschitz homeomorphism from the unit circle onto a Dini-smooth Jordan curve $\gamma$. Let $g$ be an arc-length parametrization of $\gamma$, let $\psi(t)=g^{-1}\left(F\left(e^{i t}\right)\right)$, and define $\Psi(t)=F\left(e^{i t}\right)=g(\psi(t))$. Then for almost every $\tau \in[0,2 \pi]$, the limit

$$
J_{f}\left(e^{i \tau}\right):=\lim _{r \rightarrow 1} J_{f}\left(r e^{i \tau}\right)
$$

exists and we have

$$
J_{f}\left(e^{i \tau}\right)=\psi^{\prime}(\tau) \int_{0}^{2 \pi} \frac{\operatorname{Re}\left[\overline{(g(\psi(t))-g(\psi(\tau)))} \cdot i g^{\prime}(\psi(\tau))\right]}{2 \sin ^{2}((t-\tau) / 2)} \frac{d t}{2 \pi} .
$$

From Lemma 2.2 and Lemma 2.4 we obtain

Lemma 2.5. Under the conditions and notation of Lemma 2.4 we have

$$
J_{f}\left(e^{i \varphi}\right) \leq \frac{\pi}{4}\left|\Psi^{\prime}(\varphi)\right| \int_{-\pi}^{\pi} \frac{1}{x^{2}} \int_{0}^{d_{\gamma}\left(F\left(e^{i(\varphi+x)}\right), F\left(e^{i \varphi}\right)\right)} \omega(\tau) d \tau d x
$$

for a.e. $e^{i \varphi} \in \mathbb{T}$. Here $\omega$ is the modulus of continuity of $g^{\prime}$. 
Lemma 2.6. Let $f=\mathcal{P}[F](z)$ be a harmonic mapping between the unit disk $\mathbb{U}$ and the Jordan domain $\Omega$, with $F \in C^{1, \varpi}(\mathbb{T})$. Then the partial derivatives of $f$ have a continuous extension to the boundary of the unit disk.

Proof. In the proof of this lemma we denote $\partial_{t} \Psi\left(e^{i t}\right)$ by $\Psi^{\prime}(t)$. If $F$ is Lipschitzcontinuous, then $\Phi=\Psi^{\prime} \in L^{\infty}(\mathbb{T})$, and by the famous Marcel Riesz theorem (see for example [Garnett 1981, Theorem 2.3]) there is a constant $A_{p}$ such that

$$
\left\|H\left(\Psi^{\prime}\right)\right\|_{L^{p}(\mathbb{T})} \leq A_{p}\left\|\Psi^{\prime}\right\|_{L^{p}(\mathbb{T})}
$$

for $1<p<\infty$. It follows that $\tilde{\Phi}=H\left(\Psi^{\prime}\right) \in L^{1}$. Since $r f_{r}$ is the harmonic conjugate of $f_{\tau}$, we have $r w_{r}=\mathcal{P}\left[H\left(\Psi^{\prime}\right)\right]$ according to (1-5). By again using Fatou's theorem, we have

$$
\lim _{r \rightarrow 1^{-}} f_{r}\left(r e^{i \tau}\right)=H\left(\Psi^{\prime}\right)(\tau) \quad \text { a.e. }
$$

By (1-4), and by following the proof of Privaloff's theorem [Zygmund 1959], we obtain that if $\left|\Psi^{\prime}(x)-\Psi^{\prime}(y)\right| \leq \omega(|x-y|)$ for the Dini-continuous function, then

$$
\left|H\left(\Psi^{\prime}\right)(x+h)-H\left(\Psi^{\prime}\right)(x)\right| \leq A \int_{0}^{2 h} \frac{\omega(t)}{t} d t+B h \int_{h}^{2 \pi} \frac{\omega(t)}{t^{2}} d t+C \omega(h),
$$

for some absolute constants $A, B$ and $C$. The detailed proof of the last fact can be found in [Garnett 1981, Theorem III 1.3.]. This implies that $r w_{r}\left(r e^{i t}\right)$ and $f_{t}\left(r e^{i t}\right)$ have continuous extensions to the boundary and this is what we needed to prove.

We now prove the following lemma needed in the sequel.

Lemma 2.7. Let $A$ be a positive integrable function in $[0, B]$ and assume that $q, Q>0$. Then there exists a continuous increasing function $\chi$ of $(0,+\infty)$ into itself, depending on $A, B, q$ and $Q$, such that the following hold: $\lim _{x \rightarrow \infty} \chi(x)=$ $\infty$, the function $g(x)=x \chi(x)$ is convex, and

$$
\int_{0}^{B} A(x) \chi\left(Q x^{-q}\right) d x \leq 4 \int_{0}^{B} A(x) d x .
$$

Proof. First define inductively a sequence $x_{0}=B, x_{k}>0$ for $k>0$, such that $x_{k+1}<x_{k} / 2$, and

$$
\int_{0}^{x_{k}} A(x) d x \leq M 2^{-k} \text { where } M=\int_{0}^{B} A(x) d x .
$$

This is possible because $A$ is integrable.

Then define a continuous function $\xi$ in $[0, B]$ by $\xi\left(x_{k}\right)=k$, and by extending it linearly on each interval $\left[x_{k+1}, x_{k}\right]$, that is

$$
\xi(x)=k+\frac{x_{k}-x}{x_{k}-x_{k+1}}, \quad x \in\left[x_{k+1}, x_{k}\right] .
$$


It is easy to see that this function is convex, decreasing and tends to $+\infty$ as $x \rightarrow \infty$. Moreover

$$
\int_{0}^{B} A(x) \xi(x) d x \leq M \sum_{k=0}^{\infty}(k+1) 2^{-k}=4 M .
$$

Now set $\chi(x)=\xi\left((Q / x)^{\tau}\right)$ for $\tau=1 / q$. It remains to verify that $x \chi(x)$ is convex. This we do by differentiation:

$$
(x \chi(x))^{\prime}=\xi\left(Q^{\tau} x^{-\tau}\right)-Q^{\tau} \tau x^{-\tau} \xi^{\prime}\left(Q^{\tau} x^{-\tau}\right) .
$$

Since both summands are increasing, $x \chi(x)$ is convex.

\section{The proof of Theorem 1.1}

By assumption of the theorem, the derivative of an arc-length parametrization $g^{\prime}$ has a Dini-continuous modulus of continuity $\omega$. We consider two cases.

(i) $F\left(e^{i t}\right)=\Psi(t) \in C^{1, \varpi}(\mathbb{T})$. Then by Lemma 2.6 the mapping $f(z)=\mathcal{P}[F](z)$ is $C^{1}$ up to the boundary. First we notice that for $L=\sup \left|\Psi^{\prime}(t)\right|$, it is clear that $L<\infty$. We will prove more. We will show that $L$ is bounded by a constant not depending a priori on $F$. According to Lemma 2.6 and to (1-1), we have

$$
\begin{aligned}
\left|D f\left(e^{i \varphi}\right)\right|^{2} & =\left(\left|f_{z}\left(e^{i \varphi}\right)\right|+\left|f_{\bar{z}}\left(e^{i \varphi}\right)\right|\right)^{2} \\
& =\lim _{z \rightarrow e^{i \varphi}}\left(\left|f_{z}(z)\right|+\left|f_{\bar{z}}(z)\right|\right)^{2} \\
& \leq K \lim _{z \rightarrow e^{i \varphi}}\left(\left|f_{z}(z)\right|^{2}-\left|f_{\bar{z}}(z)\right|^{2}\right) \\
& =K\left(\left|f_{z}\left(e^{i \varphi}\right)\right|^{2}-\left|f_{\bar{z}}\left(e^{i \varphi}\right)\right|^{2}\right)=K J_{f}\left(e^{i \varphi}\right) .
\end{aligned}
$$

Furthermore, we have

$$
\left|D f\left(r e^{i \varphi}\right)\right|=\sup _{|\xi|=1}\left|D f\left(r e^{i \varphi}\right) \xi\right| \geq\left|D f\left(r e^{i \varphi}\right)\left(i e^{i \varphi}\right)\right|=\left|\partial_{\varphi} f\left(r e^{i \varphi}\right)\right| .
$$

This implies that

$$
\left|D f\left(e^{i \varphi}\right)\right|^{2} \geq\left|\partial_{\varphi} f\left(e^{i \varphi}\right)\right|^{2}=\left|\Psi^{\prime}(\varphi)\right|^{2} .
$$

From (2-9), (3-3) and (3-1), we obtain:

$$
\left|\Psi^{\prime}(\varphi)\right|^{2} \leq K C_{1}\left|\Psi^{\prime}(\varphi)\right| \int_{-\pi}^{\pi} \frac{1}{x^{2}} \int_{0}^{\rho(x, \varphi)} \omega(\tau) d \tau d x,
$$

where

$$
\rho(x, \varphi)=d_{\gamma}\left(F\left(e^{i(\varphi+x)}\right), F\left(e^{i \varphi}\right)\right),
$$


which is the same as

$$
\left|\Psi^{\prime}(\varphi)\right| \leq K C_{1} \int_{-\pi}^{\pi} \frac{\rho(\varphi, x)}{x^{2}} \int_{0}^{1} \omega(\tau \rho(\varphi, x)) d \tau d x .
$$

Thus

$$
\left|\Psi^{\prime}(\varphi)\right| \leq K C_{1} \int_{-\pi}^{\pi} \frac{\rho(\varphi, x)}{x^{2}} \omega(\rho(\varphi, x)) d x
$$

Let

$$
L:=\max _{x \in[0,2 \pi]}\left|\Psi^{\prime}(x)\right|=\max _{x \in[0,2 \pi]} \psi^{\prime}(x)=\psi^{\prime}(\varphi)
$$

Then

$$
L \leq K C_{1} \int_{-\pi}^{\pi} \frac{\rho(\varphi, x)}{x^{2}} \omega(\rho(\varphi, x)) d x .
$$

Furthermore, we have

$$
M:=\frac{L}{2 \pi K C_{1}} \leq \int_{-\pi}^{\pi} M(x, \varphi) \frac{d x}{2 \pi},
$$

where

$$
M(x, \varphi)=\frac{\rho(\varphi, x)}{x^{2}} \omega(\rho(\varphi, x)) .
$$

The idea is to make use of Lemma 2.7 with a convex function depending only on $K$ to be found below.

Assume that $\chi: \mathbb{R}^{+} \rightarrow \mathbb{R}^{+}$is a continuous increasing function to be determined in the sequel such that the function $\Phi(t)=t \chi(t)$ is convex. By using Jensen's inequality to the previous integral with respect to the convex function $\Phi$, we obtain

$$
\Phi(M) \leq \int_{-\pi}^{\pi} \Phi(M(x, \varphi)) \frac{d x}{2 \pi},
$$

or equivalently,

$$
M \chi(M) \leq \int_{-\pi}^{\pi} M(x, \varphi) \chi(M(x, \varphi)) \frac{d x}{2 \pi} .
$$

From (2-7) and (3-4) we deduce that

$$
\rho(\varphi, x) \leq B_{\gamma} L|x| .
$$

On the other hand, since $f$ is a normalized qc mapping, we have by Lemma 2.3 that

$$
\rho(\varphi, x) \leq B_{\gamma} \Lambda_{\gamma}(K)|x|^{\alpha} .
$$


Notice that this time we used the boundary normalization. This implies that

$$
M(x, \varphi)=\frac{\rho(\varphi, x)}{x^{2}} \omega(\rho(\varphi, x)) \leq \frac{B_{\gamma} L}{x} \omega\left(B_{\gamma} \Lambda_{\gamma}(K)|x|^{\alpha}\right),
$$

and

$$
M(x, \varphi)=\frac{\rho(\varphi, x)}{x^{2}} \omega(\rho(\varphi, x)) \leq \frac{B_{\gamma} \Lambda_{\gamma}(K)}{x^{2-\alpha}} \omega\left(B_{\gamma} \Lambda_{\gamma}(K)|x|^{\alpha}\right) .
$$

So, in view of Definition 2.1 we have

$$
M(x, \varphi) \leq \frac{B_{\gamma} \Lambda_{\gamma}(K)}{x^{2-\alpha}} \omega(|\gamma|) .
$$

From (3-5) and (3-8), we obtain

$$
\begin{aligned}
\chi\left(\frac{L}{2 \pi K C_{1}}\right) & \leq \int_{-\pi}^{\pi} \frac{K C_{1} B_{\gamma}}{x} \omega\left(B_{\gamma} \Lambda_{\gamma}(K)|x|^{\alpha}\right) \chi\left(\frac{B_{\gamma} \Lambda_{\gamma}(K) \omega(|\gamma|)}{|x|^{2-\alpha}}\right) d x \\
& =2 \int_{0}^{\pi} \frac{K C_{1} B_{\gamma}}{x} \omega\left(B_{\gamma} \Lambda_{\gamma}(K)|x|^{\alpha}\right) \chi\left(\frac{B_{\gamma} \Lambda_{\gamma}(K) \omega(|\gamma|)}{|x|^{2-\alpha}}\right) d x \\
& =\frac{2 K C_{1} B_{\gamma}}{B_{\gamma} \Lambda_{\gamma}(K) \alpha} \int_{0}^{B} \frac{\omega(y)}{y} \chi\left(Q y^{1-2 / \alpha}\right) d y
\end{aligned}
$$

where

$$
B=B_{\gamma} \Lambda_{\gamma}(K) \pi^{\alpha} \quad \text { and } \quad Q=\omega(|\gamma|)\left(B_{\gamma} \Lambda_{\gamma}(K)\right)^{2-2 / \alpha} .
$$

In view of the last term of (3-11), now is the time to determine the function $\chi$. Lemma 2.7 with $q=2 / \alpha-1$ and $A(y)=\omega(y) / y$, provides us with a function $\chi$ such that $\Phi$ is convex and such that the estimate

$$
\int_{0}^{B} \frac{\omega(y)}{y} \chi\left(Q y^{1-2 / \alpha}\right) d y \leq 4 \int_{0}^{B} \frac{\omega(y)}{y} d y
$$

holds. From (3-11), we have

$$
\chi\left(\frac{L}{2 \pi K C_{1}}\right) \leq \frac{8 K C_{1} B_{\gamma}}{B_{\gamma} \Lambda_{\gamma}(K) \alpha} \int_{0}^{B} \frac{\omega(y)}{y} d y=: \Upsilon(K, \Omega) .
$$

Since $\chi$ is increasing, we infer finally that

$$
L \leq 2 \pi K C_{1} \cdot \chi^{-1}(\Upsilon(K, \Omega))=\frac{\pi^{2}}{2} K \cdot \chi^{-1}(\Upsilon(K, \Omega)) .
$$

By the maximum principle, for $z=r e^{i \varphi}$, we further have

$$
\left|\partial_{\varphi} f(z)\right| \leq L .
$$

Since $f$ is $K$-quasiconformal, we have

$$
|D w(z)| \leq K\left|\partial_{\varphi} f(z)\right| .
$$


This and the mean value inequality imply that

$$
\left|f(z)-f\left(z^{\prime}\right)\right| \leq K L\left|z-z^{\prime}\right|, \quad|z|<1,\left|z^{\prime}\right|<1 .
$$

(ii) $F \notin C^{1, \varpi}(\mathbb{T})$. In order to deal with nonsmooth $F$, we make use of an approximation argument. We begin by this definition.

Definition 3.1. Let $G$ be a domain in $\mathbb{C}$ and let $a \in \partial G$. We will say that $G_{a} \subset G$ is a neighborhood of $a$ if there exists a disk $D(a, r):=\{z:|z-a|<r\}$ such that $(D(a, r) \cap G) \subset G_{a}$.

Let $t=e^{i x} \in \mathbb{T}$. Then $F(t)=\Psi(x) \in \partial \Omega$. Let $g$ be an arc-length parametrization of $\partial \Omega$ with $g(\psi(x))=F\left(e^{i x}\right)$, where $\psi:[0,2 \pi] \rightarrow[0,|\gamma|]$ is as in the first part of the proof. Put $s=\psi(x)$. Since the modulus of continuity of $g^{\prime}$ is a Dini-continuous function $\omega$, there exists a neighborhood $\Omega_{t}$ of $\Psi(t)$ such that the derivative of its arc-length parametrization $g_{t}^{\prime}$ has modulus of continuity $C_{t} \cdot \omega$. Moreover, there exist positive numbers $r_{t}$ and $R_{t}$ such that

$$
\begin{aligned}
& \Omega_{t}^{\tau}:=\Omega_{t}+i g^{\prime}(s) \cdot \tau \subset \Omega, \quad \tau \in\left(0, R_{t}\right), \\
& \partial \Omega_{t}^{\tau} \subset \Omega, \quad \tau \in\left(0, R_{t}\right) \\
& g\left[s-r_{t}, s+r_{t}\right] \subset \partial \Omega_{t} .
\end{aligned}
$$

An example of a family $\Omega_{t}^{\tau}$ such that $\partial \Omega_{t}^{\tau} \in C^{1, \alpha}$ for $0<\alpha<1$ with property (3-14) has been given in [Kalaj 2008]. The same construction yields the family $\partial \Omega_{t}^{\tau}$ with the above mentioned properties.

Take $U_{\tau}=f^{-1}\left(\Omega_{t}^{\tau}\right)$. Let $\eta_{t}^{\tau}$ be a conformal mapping of the unit disk onto $U_{\tau}$ with normalized boundary condition: $\eta_{t}^{\tau}\left(e^{i 2 k \pi / 3}\right)=f^{-1}\left(\zeta_{k}\right)$ for $k=0,1,2$, where $\zeta_{0}, \zeta_{1}, \zeta_{2}$ are three points of $\partial \Omega_{t}^{\tau}$ of equal distance. Then the mapping

$$
f_{t}^{\tau}(z):=f\left(\eta_{t}^{\tau}(z)\right)-i g^{\prime}(s) \cdot \tau
$$

is a harmonic $K$-quasiconformal mapping of the unit disk onto $\Omega_{t}$ satisfying the boundary normalization. Moreover,

$$
f_{t}^{\tau}=\mathcal{P}\left[F_{t}^{\tau}\right] \in C^{1}(\overline{\mathbb{U}})
$$

for some function $F_{t}^{\tau} \in C^{1}(\mathbb{T})$.

Since $[0, l]$ is compact, there exists a finite family of Jordan arcs

$$
\gamma_{j}=g\left(s_{j}-r_{s_{j}} / 2, s_{j}+r_{s_{j}} / 2\right), \quad j=1, \ldots, n,
$$

covering $\gamma$. Assume that $F\left(t_{j}\right)=s_{j}$. Let

$$
F_{j, \tau}:=F_{t_{j}}^{\tau}, \quad a_{j, \tau}:=\eta_{t_{j}}^{\tau} \quad \text { and } \quad f_{j, \tau}:=f_{t_{j}}^{\tau} .
$$


Using the case $F \in C^{1, \varpi}$, it follows that there exists a constant $C_{j}^{\prime}=C^{\prime}\left(K, \gamma_{j}\right)$ such that

$$
\left|\partial_{\varphi} F_{j, \tau}^{\prime}\left(e^{i \varphi}\right)\right| \leq C_{j}^{\prime}
$$

and

$$
\left|f_{j, \tau}\left(z_{1}\right)-f_{j, \tau}\left(z_{2}\right)\right| \leq K C_{j}^{\prime}\left|z_{1}-z_{2}\right| .
$$

Since $a_{j, \tau}(z)$ converges uniformly on compact subsets of $\mathbb{U}$ to the function $a_{j, 0}(z)$ when $\tau \rightarrow 0$, and since $f_{j, \tau}=f \circ a_{j, \tau}$, inequality (3-17) implies

$$
\left|f_{j}\left(z_{1}\right)-f_{j}\left(z_{2}\right)\right| \leq K C_{j}^{\prime}\left|z_{1}-z_{2}\right| \text { for } z_{1}, z_{2} \in \overline{\mathbb{U}},
$$

where $f_{j}=f \circ a_{j, 0}=\mathcal{P}\left[F_{j}\right]$. For $z_{1}=e^{i t}$ and $z_{2}=e^{i \varphi}$ for $t \rightarrow \varphi$, we obtain that $\left|\partial_{\varphi} F_{j}\left(e^{i \varphi}\right)\right| \leq K C_{j}^{\prime}$ a.e. Since the mapping $b_{j}=a_{0, j}^{-1}$ can be extended conformally across the $\operatorname{arc} S_{j}=f^{-1}\left(\lambda_{j}\right)$, where $\lambda_{j}=g\left(s_{j}-t_{s_{j}}, s_{j}+t_{s_{j}}\right)$, there exists a constant $L_{j}$ such that $\left|b_{j}(z)\right| \leq L_{j}$ on $S_{j}^{\prime}=\mathbb{T} \cap f^{-1}\left(\gamma_{j}\right)$ for $j=1, \ldots, n$. Hence $\left|\partial_{\varphi} F\left(e^{i \varphi}\right)\right| \leq$ $K C_{j}^{\prime} \cdot L_{j}$ on $S_{j}^{\prime}$. Let $C^{\prime}=\max \left\{K C_{j}^{\prime} \cdot L_{j}: j=1, \ldots, n\right\}$. Inequalities (1-7) and (1-8) easily follow from $\mathbb{T}=\bigcup_{j=1}^{n} S_{j}^{\prime}$.

Notice that we can now repeat the first part of the proof for a Lipschitz $f=\mathcal{P}[F]$ in order to obtain a more concrete Lipschitz constant, i.e., the constant $L$ satisfying (3-12). The proof is complete.

\section{Acknowledgement}

I am grateful to professor Alexandre Eremenko, whose idea is used in the proof of Lemma 2.7, for his numerous useful suggestions and corrections that have substantially improved this paper. I am grateful to the anonymous referee for some useful suggestions and corrections.

\section{References}

[Axler et al. 1992] S. Axler, P. Bourdon, and W. Ramey, Harmonic function theory, Graduate Texts in Mathematics 137, Springer, New York, 1992. MR 93f:31001 Zbl 0765.31001

[Choquet 1945] G. Choquet, "Sur un type de transformation analytique généralisant la représentation conforme et définie au moyen de fonctions harmoniques", Bull. Sci. Math. (2) 69 (1945), 156-165. MR 8,93a Zbl 0063.00851

[Duren 1970] P. L. Duren, Theory of $H^{p}$ spaces, Pure and Applied Mathematics 38, Academic Press, New York, 1970. MR 42 \#3552 Zbl 0215.20203

[Duren 2004] P. Duren, Harmonic mappings in the plane, Cambridge Tracts in Mathematics 156, Cambridge Univ. Press, 2004. MR 2005d:31001 Zbl 1055.31001

[Duren and Hengartner 1997] P. Duren and W. Hengartner, "Harmonic mappings of multiply connected domains", Pacific J. Math. 180:2 (1997), 201-220. MR 99f:30051 Zbl 0885.30020

[Garnett 1981] J. B. Garnett, Bounded analytic functions, Pure and Applied Mathematics 96, Academic Press, New York, 1981. MR 83g:30037 Zbl 0469.30024 
[Goluzin 1969] G. M. Goluzin, Geometric theory of functions of a complex variable, Translations of Mathematical Monographs 26, Amer. Math. Soc., Providence, RI, 1969. MR 40 \#308 Zbl 0183.07502

[Jost 1981] J. Jost, "Univalency of harmonic mappings between surfaces", J. Reine Angew. Math. 324 (1981), 141-153. MR 82h:58013 Zbl 0453.53036

[Kalaj 2003] D. Kalaj, "On harmonic diffeomorphisms of the unit disc onto a convex domain", Complex Var. Theory Appl. 48:2 (2003), 175-187. MR 2003m:30043 Zbl 1041.30006

[Kalaj 2004] D. Kalaj, "Quasiconformal harmonic functions between convex domains", Publ. Inst. Math. (Beograd) (N.S.) 76:90 (2004), 3-20. MR 2005j:30032 Zbl 1220.30032

[Kalaj 2008] D. Kalaj, "Quasiconformal and harmonic mappings between Jordan domains", Math. Z. 260:2 (2008), 237-252. MR 2009e:30038 Zbl 1151.30014

[Kalaj 2011a] D. Kalaj, "Harmonic mappings and distance function", Ann. Sc. Norm. Super. Pisa Cl. Sci. (5) 10:3 (2011), 669-681. MR 2012j:30054 Zbl 1252.30018

[Kalaj 2011b] D. Kalaj, "Invertible harmonic mappings beyond the Kneser theorem and quasiconformal harmonic mappings", Studia Math. 207:2 (2011), 117-136. MR 2012k:30065 Zbl 1279.30032

[Kalaj 2012] D. Kalaj, "On boundary correspondences under quasiconformal harmonic mappings between smooth Jordan domains”, Math. Nach. 285:2-3 (2012), 283-294. MR 2881282 Zbl 1251.30032

[Kalaj 2013] D. Kalaj, "A priori estimate of gradient of a solution of a certain differential inequality and quasiconformal mappings", J. Anal. Math. 119:1 (2013), 63-88. MR 3043147 Zbl 1270.35148

[Kalaj and Mateljević 2006] D. Kalaj and M. Mateljević, "Inner estimate and quasiconformal harmonic maps between smooth domains", J. Anal. Math. 100 (2006), 117-132. MR 2008b:30033 Zbl 1173.30311

[Kellogg 1912] O. D. Kellogg, "Harmonic functions and Green's integral", Trans. Amer. Math. Soc. 13:1 (1912), 109-132. MR 1500909 JFM 43.0889.01

[Lesley and Warschawski 1978] F. D. Lesley and S. E. Warschawski, "On conformal mappings with derivative in VMOA”, Math. Z. 158:3 (1978), 275-283. MR 57 \#6393 Zbl 0374.30007

[Lewy 1936] H. Lewy, "On the non-vanishing of the Jacobian in certain one-to-one mappings", Bull. Amer. Math. Soc. 42:10 (1936), 689-692. MR 1563404 Zbl 0015.15903

[Martio 1968] O. Martio, "On harmonic quasiconformal mappings", Ann. Acad. Sci. Fenn. Ser. A I No. 425 (1968), 10. MR 38 \#4678 Zbl 0162.37902

[Pavlović 2002] M. Pavlović, "Boundary correspondence under harmonic quasiconformal homeomorphisms of the unit disk", Ann. Acad. Sci. Fenn. Math. 27:2 (2002), 365-372. MR 2003g:30030 Zbl 1017.30014

[Pavlović 2004] M. Pavlović, Introduction to function spaces on the disk, Posebna Izdanja 20, Matematički Institut SANU, Belgrade, 2004. MR 2006d:30001 Zbl 1107.30001

[Pommerenke 1975] C. Pommerenke, Univalent functions, Studia Mathematica/Mathematische Lehrbücher 25, Vandenhoeck \& Ruprecht, Göttingen, 1975. MR 58 \#22526 Zbl 0298.30014

[Warschawski 1961] S. E. Warschawski, "On differentiability at the boundary in conformal mapping", Proc. Amer. Math. Soc. 12 (1961), 614-620. MR 24 \#A1374 Zbl 0100.28803

[Warschawski 1970] S. E. Warschawski, "Boundary derivatives of minimal surfaces", Arch. Rational Mech. Anal. 38 (1970), 241-256. MR 41 \#7549 Zbl 0209.41802

[Zygmund 1959] A. Zygmund, Trigonometric series, I, 2nd ed., Cambridge Univ. Press, 1959. MR 21 \#6498 Zbl 0085.05601

Received February 7, 2014. Revised December 17, 2014. 
DAVID KALAJ

FACUlty of Natural SCIENCES ANd Mathematics UNIVERSITY OF MONTENEGRO

DZORDZA VASINGTONA B.B.

81000 PODGORICA

MONTENEGRo

davidk@ac.me 


\title{
PACIFIC JOURNAL OF MATHEMATICS
}

\author{
msp.org/pjm
}

Founded in 1951 by E. F. Beckenbach (1906-1982) and F. Wolf (1904-1989)

\section{EDITORS}

Don Blasius (Managing Editor)

Department of Mathematics

University of California

Los Angeles, CA 90095-1555

blasius@math.ucla.edu

\author{
Paul Balmer \\ Department of Mathematics \\ University of California \\ Los Angeles, CA 90095-1555 \\ balmer@math.ucla.edu \\ Robert Finn \\ Department of Mathematics \\ Stanford University \\ Stanford, CA 94305-2125 \\ finn@math.stanford.edu \\ Sorin Popa \\ Department of Mathematics \\ University of California \\ Los Angeles, CA 90095-1555 \\ popa@math.ucla.edu
}

\author{
Vyjayanthi Chari \\ Department of Mathematics \\ University of California \\ Riverside, CA 92521-0135 \\ chari@math.ucr.edu \\ Kefeng Liu \\ Department of Mathematics \\ University of California \\ Los Angeles, CA 90095-1555 \\ liu@math.ucla.edu \\ Jie Qing \\ Department of Mathematics \\ University of California \\ Santa Cruz, CA 95064 \\ qing@ cats.ucsc.edu
}

\section{PRODUCTION}

Silvio Levy, Scientific Editor, production@msp.org

\section{SUPPORTING INSTITUTIONS}

ACADEMIA SINICA, TAIPEI

CALIFORNIA INST. OF TECHNOLOGY

INST. DE MATEMÁTICA PURA E APLICADA

KEIO UNIVERSITY

MATH. SCIENCES RESEARCH INSTITUTE

NEW MEXICO STATE UNIV.

OREGON STATE UNIV.

\author{
STANFORD UNIVERSITY \\ UNIV. OF BRITISH COLUMBIA \\ UNIV. OF CALIFORNIA, BERKELEY \\ UNIV. OF CALIFORNIA, DAVIS \\ UNIV. OF CALIFORNIA, LOS ANGELES \\ UNIV. OF CALIFORNIA, RIVERSIDE \\ UNIV. OF CALIFORNIA, SAN DIEGO \\ UNIV. OF CALIF., SANTA BARBARA
}

\author{
Daryl Cooper \\ Department of Mathematics \\ University of California \\ Santa Barbara, CA 93106-3080 \\ cooper@math.ucsb.edu \\ Jiang-Hua Lu \\ Department of Mathematics \\ The University of Hong Kong \\ Pokfulam Rd., Hong Kong \\ jhlu@maths.hku.hk \\ Paul Yang \\ Department of Mathematics \\ Princeton University \\ Princeton NJ 08544-1000 \\ yang@math.princeton.edu
}

These supporting institutions contribute to the cost of publication of this Journal, but they are not owners or publishers and have no responsibility for its contents or policies.

See inside back cover or msp.org/pjm for submission instructions.

The subscription price for 2015 is US \$420/year for the electronic version, and \$570/year for print and electronic.

Subscriptions, requests for back issues and changes of subscribers address should be sent to Pacific Journal of Mathematics, P.O. Box 4163, Berkeley, CA 94704-0163, U.S.A. The Pacific Journal of Mathematics is indexed by Mathematical Reviews, Zentralblatt MATH, PASCAL CNRS Index, Referativnyi Zhurnal, Current Mathematical Publications and Web of Knowledge (Science Citation Index).

The Pacific Journal of Mathematics (ISSN 0030-8730) at the University of California, c/o Department of Mathematics, 798 Evans Hall \#3840, Berkeley, CA 94720-3840, is published twelve times a year. Periodical rate postage paid at Berkeley, CA 94704, and additional mailing offices. POSTMASTER: send address changes to Pacific Journal of Mathematics, P.O. Box 4163, Berkeley, CA 94704-0163.

PJM peer review and production are managed by EditFLOW ${ }^{\circledR}$ from Mathematical Sciences Publishers.

\section{PUBLISHED BY}

\section{mathematical sciences publishers \\ nonprofit scientific publishing}

http://msp.org/

(C) 2015 Mathematical Sciences Publishers 


\section{PACIFIC JOURNAL OF MATHEMATICS}

Volume $276 \quad$ No. $1 \quad$ July 2015

On the degree of certain local $L$-functions

U. K. ANANDAVARdhanan and AmiYa Kumar MondaL

Torus actions and tensor products of intersection cohomology

ASILATA BAPAT

Cyclicity in Dirichlet-type spaces and extremal polynomials II: functions on the bidisk

Catherine Bénéteau, Alberto A. Condori, Constanze Liaw,

DANIEl SECo and Alan A. SOlA

Compactness results for sequences of approximate biharmonic maps

CHRISTINE BREINER and TOBIAS LAMM

Criteria for vanishing of Tor over complete intersections

Olgur Celikbas, Srikanth B. Iyengar, Greg PiePMeyer and

ROGER WIEGAND

Convex solutions to the power-of-mean curvature flow

\section{SHIBING CHEN}

Constructions of periodic minimal surfaces and minimal annuli in $\mathrm{Sol}_{3}$

CHRISTOPHE DESMONTS

Quasi-exceptional domains

ALEXANDRE EREMENKO and ERIK LUNDBERG

Endoscopic transfer for unitary groups and holomorphy of Asai $L$-functions

NEVEN GRBAC and FREYDOON SHAHIDI

Quasiconformal harmonic mappings between Dini-smooth Jordan domains

DAVID KALAJ

Semisimple super Tannakian categories with a small tensor generator

THOMAS KRÄMER and RAINER WEISSAUER

On maximal Lindenstrauss spaces

PETR PETRÁČEK and JiŘí SPURNÝ 